\title{
Hirschsprung's Disease and Congenital Deafness
}

\author{
ROSALIND SKINNER and DAVID IRVINE
}

\author{
The University Department of Human Genetics, Western General Hospital, Edinburgh
}

\begin{abstract}
Summary. An association of Hirschsprung's disease (congenital intestinal aganglionosis) and profound congenital deafness is described in four unrelated patients and another reported case which was found in the literature. It is suggested that this might represent a new syndrome.
\end{abstract}

Hirschsprung's disease (megacolon or congenital intestinal aganglionosis) is believed to be inherited on a multifactorial basis, with a higher familial incidence among the relatives of patients with a long aganglionic segment (Bodian and Carter, 1962/1963; Passarge, 1967). However, profound childhood deafness, when not due to any exogenous factor, is usually inherited as an autosomal recessive trait. It has been estimated that about $90 \%$ of cases of profound childhood deafness, exhibiting simple Mendelian inheritance, are autosomal recessive in nature (Fraser, 1964). The present communication concerns a report of four patients with Hirschsprung's disease who also had profound childhood deafness.

\section{Case Reports}

Three of the reported cases were found during a survey of children with profound deafness who were attending a school for deaf children. The fourth case was ascertained from amongst a series of children who had been operated upon for Hirschsprung's disease.

Case 1. (C.K.) This 16-year-old boy was the product of a full-term, normal delivery which followed an uneventful pregnancy. $\mathrm{He}$ was admitted to hospital at the age of one month for investigation into the cause of intermittent vomiting which had begun on the second day of life, together with chronic constipation which had been present from birth. A tentative diagnosis of Hirschsprung's disease was made on clinical and radiological findings and he was managed conservatively at home with aperients for the next two years. However, repeated bouts of abdominal distention associated with faecal retention occurred and necessitated further investigation. A rectal biopsy was performed and histological examination revealed an absence of ganglion cells confirming the diagnosis of Hirschsprung's disease.

Received 18 May 1973.
At the age of 27 months he underwent a rectosigmoidectomy, which subsequently proved to be successful. At the age of nine months the parents had begun to suspect that he was deaf. This impression was confirmed when the patient was 18 months old, before he had any treatment for his bowel condition other than aperients.

On clinical examination no abnormalities were detected except for a mild convergent strabismus. In particular there was no evidence of any pigmentary anomaly or of any neurological involvement.

Apart from his Hirschsprung's disease and deafness, he has a marked behavioural disorder requiring psychiatric attention and is of borderline normal intelligence.

His parents are unrelated and he has one brother six years older than himself who is quite well. There is no family history of either deafness or Hirschsprung's disease.

Case 2. (A. McT.) This 20-year-old male was the product of a full-term, normal delivery. His mother suffered from a severe attack of mumps at about 34 weeks of pregnancy. She was not given any antibiotics and apart from this the pregnancy was uncomplicated. Within the first few days of life the patient presented with feeding difficulties, intermittent vomiting, and constipation. At the age of seven weeks a tentative diagnosis of Hirschsprung's disease was made on clinical and radiological grounds. Because of repeated attacks of vomiting and faecal retention he underwent a laparotomy at the age of five months and a defunctioning colostomy was performed. Histological examination of sections of bowel obtained at operation confirmed the diagnosis of Hirschsprung's disease. Just before the operation the patient received a short course of streptomycin because of unexplained persistent pyrexia. At the age of two years he underwent a definitive operation for the removal of the aganglionic segment of bowel (rectosigmoidectomy).

The patient was first noticed to be deaf between one and two years of age and this was confirmed just after he was two years old. Apart from his Hirschsprung's disease and deafness, there were no other associated 
physical anomalies on clinical examination. In particular there was no evidence of any pigmentary anomaly or any neurological involvement.

His parents are unrelated and he has a brother and two sisters all of whom are well. There is no family history of either Hirschsprung's disease or deafness.

Case 3. G.M. is a 16-year-old boy about whom only a limited amount of information is available. He underwent a Rammstedt operation at the age of six weeks because of pyloric stenosis. In view of a history of chronic constipation he was suspected of also having Hirschsprung's disease. This diagnosis was confirmed and he was treated surgically at about three years of age. It appears that deafness was present from infancy and was probably congenital.

There were no significant abnormalities on physical examination but he is mentally retarded and was in fact admitted to an institution for the mentally subnormal on leaving the school for deaf children.

His parents are unrelated and he has four brothers and two sisters all of whom are well. There is no family history of deafness or Hirschsprung's disease.

Case 4. (T.T.) This 18-year-old boy was the product of a full-term, normal delivery which followed an uneventful pregnancy. He presented with chronic constipation in the neonatal period and was diagnosed as having Hirschsprung's disease. He then developed projectile vomiting at about six months of age. He continued to be managed conservatively however until about three years of age. At this time he was reinvestigated because of a deterioration in his condition and histological examination of a rectal biopsy confirmed the diagnosis of Hirschsprung's disease. A colostomy was then performed and the operation was followed by an attack of peritonitis which was treated with streptomycin. About six months later he underwent a rectosigmoidectomy. There followed many complications requiring surgical treatment and frequent courses of antibiotics.

The patient was first noted to be deaf at about five and a half years of age although his mother felt she had noticed it as early as two years of age. At the age of seven years he was confirmed as having a moderately severe degree of nerve deafness, particularly in the higher frequency range.

Clinical examination revealed no associated physical abnormalities, in particular there was no evidence of any pigmentary anomalies or of any neurological involvement.

His parents are unrelated and there is no family history of either deafness or Hirschsprung's disease.

\section{Discussion}

Four patients in whom there was an association of Hirschsprung's disease and deafness are described. In all cases the deafness appeared to be neural in origin. In reviewing the literature this association was found to be recorded in three cases. Bodian and Carter (1962/1963) quote two cases in their patients with abnormalities associated with Hirschsprung's disease. One case had deafness and cleft palate together with Hirschsprung's disease, whilst the other case had deafness and congenital cataract. In neither of these cases is the type of deafness defined. One other case is referred to by McKusick (1971) in his catalogue (no. 24920) in which heterochromia iridis was associated with megacolon and congenital deafness.

Hirschsprung's disease could be associated with childhood deafness in cases who received streptomycin early in the course of the disease, since this drug is now known to be ototoxic. However, in only one of the four cases reported here was streptomycin or any other antibiotic instituted before the deafness was first observed. Only in case 4 is there convincing evidence that streptomycin may have played a part in the aetiology of the patient's deafness.

Alternatively the association of Hirschsprung's disease and deafness might be fortuitous, but this seems unlikely. The incidence of Hirschsprung's disease is about 1:5000 (Passarge, 1967) whereas the incidence of profound childhood deafness is about 1:1000 (Fraser, 1964). Therefore an association of these two disorders purely by chance would be expected to occur about once in every five million births.

A possible explanation for an association between Hirschsprung's disease and deafness might be that of a common aetiological mechanism. Yntema and Hammond (1954) demonstrated, in studies on chick embryos, that the intrinsic innervation of the lower bowel originated from cells in the cervical region of the neural crest. Embryological studies on human embryos and fetuses by Okamoto and Ueda (1967) agreed with these findings and gave further support to the theory that the neuroblasts of the intramural plexus of the intestine originate in the central nervous system in humans and migrate to the alimentary tract during early embryological development. Okamoto and Ueda (1967) conclude that aganglionosis is a developmental anomaly resulting from an interruption of the migration of neural crest cells along the alimentary tract sometime before the 12th week of gestation. Similarly embryological evidence suggests that neural crest cells play an important role in the determination of the auditory organ (Fisch, 1959). Thus it appears that a common aetiological basis for Hirschsprung's disease and neural deafness may well exist.

All the cases described here are sporadic and there is no history of consanguinity in any of the families. A genetic basis for the association of Hirschsprung's disease and deafness therefore remains to be defined. 
We are very grateful to $\mathrm{Mr}$ F. H. Robarts for referring patient T.T. to us and allowing us full access to information on patients C.K. and A.McT. who were at one time under his care. We are also grateful to $\mathrm{Mr}$ W. Jeffrey, Principal of Donaldson's School for the Deaf, for his kind cooperation, and to Professor A. E. H. Emery for his help and encouragement.

\section{REFERENCES}

Bodian, M. and Carter, C. O. (1962/1963). A family study of Hirschsprung's disease. Annals of Human Genetics, 26, 261-271.
Fisch, L. (1959). Deafness as part of an hereditary syndrome. fournal of Laryngology and Otology, 73, 355-382.

Fraser, G. R. (1964). Profound childhood deafness. Fournal of Medical Genetics, 1, 118-151.

McKusick, V. A. (1971). Mendelian Inheritance in Man, 3rd edition. Johns Hopkins Press, Baltimore.

Okamoto, E. and Ueda, T. (1967). Embryogenesis of intramural ganglia of the gut and its relation to Hirschsprung's disease. fournal of Pediatric Surgery, 2, 437-443.

Passarge, E. (1967). The genetics of Hirschsprung's disease. Evidence for heterogeneous etiology and a study of sixty-three families. New England fournal of Medicine, 276, 138-143.

Yntema, C. L. and Hammond, W. L. (1954). The origin of intrinsic ganglia of trunk viscera from vagal neural crest in the chick embryo. Fournal of Comparative Neurology, 101, 515-541. 